\title{
Fundus autofluorescence in the evaluation of diabetic macular edema treatment response
}

\author{
Sergio E. Hernández-Da Mota, ${ }^{*}$ Francisco Béjar-Cornejo, ${ }^{1}$ Virgilio Lima-Gómez² and \\ Ernesto Rodríguez-Ayala ${ }^{3}$ \\ ${ }^{1}$ Retina Department, Clínica David, Morelia, Michoacán; ${ }^{2}$ Facultad de Medicina "Dr. Ignacio Chávez", Universidad Michoacana de "San Nicolás de \\ Hidalgo", Morelia, Michoacán; '30phthalmology Department, Hospital Juárez de México, Mexico City. Mexico
}

\begin{abstract}
Introduction: Fundus autofluorescence (FAF) has shown sensitivity in the detection of macular edema. Objectives: To evaluate indices formed with FAF and retinal anatomical-functional variables in patients with diabetic macular edema (DME) treated with ziv-aflibercept (ziv-AFL). Methods: Twenty-nine eyes of 15 DME patients who received ziv-AFL intravitreal injections were included in the study. Best-corrected visual acuity (BCVA), contrast sensitivity (CS), optical coherence tomography (OCT) and FAF were evaluated before treatment and at one and two months. OCT variables were central subfield thickness (CST), macular volume (MV) and macular cube average thickness (MCAT). FAF/BCVA, FAF/CS, FAF/CST, FAF/MV and AF/MCAT indices baseline values were obtained. Analysis was performed with Spearman's rank correlation coefficient and linear regression analysis. Results: There was a significant correlation between baseline FAF/BCVA index and BCVA at second month $\left(r_{s}=-0.78\right.$, $p=0.000)$, between baseline FAF/CS index and BCVA at second month $\left(r_{s}=-0.68, p=0.0009\right)$ and between baseline FAF/ $C S$ index and $M V$ at first month of follow-up $\left(r_{s}=0.64, p=0.002\right)$. Conclusions: In DME, composite indices with baseline FAF predict variables such as BCVA in the follow-up of patients receiving ziv-AFL.
\end{abstract}

KEY WORDS: Diabetic macular edema. Fundus autofluorescence. SD-OCT. Ziv-aflibercept. Intravitreal anti-VEGF therapy.

\section{Autofluorescencia retiniana en la evaluación de la respuesta al tratamiento del edema macular diabético}

\section{Resumen}

Introducción: La autofluorescencia retiniana (AF) ha mostrado sensibilidad en la detección del edema macular. Objetivos: Evaluar índices formados con la AF y variables anatomofuncionales retinianas en pacientes con edema macular diabético (EMD) tratados con ziv-aflibercept (ziv-AFL). Métodos: Fueron incluidos 29 ojos de 15 pacientes con EMD que recibieron inyecciones intravítreas de ziv-AFL. Se evaluó agudeza visual mejor corregida (AVMC), sensibilidad al contraste (SC), tomografía de coherencia óptica (TCO) y AF, antes del tratamiento, así como al primer y segundo mes de iniciado este. Las variables de la TCO fueron grosor foveal central (GFC), volumen macular (VM) y grosor promedio macular (GPM). Se obtuvieron los valores basales de AF/AVMC, AF/SC, AF/GFC, AFNM y AF/GPM. Se realizó análisis con el coeficiente de correlación de rangos de Spearman y análisis de regresión lineal. Resultados: Hubo una correlación significativa entre el índice AF/AVMC basal y la AVMC en el segundo mes $\left(r_{s}=-0.78, p=0.000\right)$, entre el índice AF/SC basal y la AVMC en el segundo mes $\left(r_{s}=-0.68, p=0.0009\right)$ y entre AF/SC basal y el VM en el primer mes de seguimiento $\left(r_{s}=0.64, p=0.002\right)$. Conclusiones: En el EMD, los índices compuestos con AF basales predicen variables como AVMC en el seguimiento de pacientes que reciben ziv-AFL.

PALABRAS CLAVE: Edema macular diabético. Autofluorescencia retiniana. Tomografia de coherencia óptica de dominio espectral. Ziv-aflibercept. Terapia anti-VEGF intravítrea.

\footnotetext{
Correspondence:

Date of reception: $21-11-2020$

*Sergio E. Hernández-Da Mota

Date of acceptance: 08-12-2020

Gac Med Mex. 2021;157:397-403

Contents available at PubMed

E-mail: tolodamota@yahoo.com.mx

DOI: 10.24875/GMM.M21000581

www.gacetamedicademexico.com

0016-3813/@ 2021 Academia Nacional de Medicina de México, A.C.. Published by Permanyer. This is an open access article under the CC BY-NC-ND license (http://creativecommons.org/licenses/by-nc-nd/4.0/).
} 


\section{Introduction}

Diabetic macular edema (DME) is the main cause of visual loss in diabetic patients. ${ }^{1,2}$ It affects approximately $30 \%$ of patients with more than 20 years of suffering from diabetes mellitus. ${ }^{3}$ Slit lamp biomicroscopy, fluorescein angiography and optical coherence tomography (OCT) are methods that have been proven useful in the diagnosis and follow-up of patients with DME. ${ }^{4,5}$

Fundus autofluorescence (FAF) is a diagnostic modality used for comprehensive evaluation of several retinal pathologies, ${ }^{6}$ which has shown certain sensitivity in the detection of cystic macular edema (CME), mainly with a short wavelength. ${ }^{7}$

Therefore, FAF may represent a non-invasive imaging technique for evaluating the response to intravitreal antiangiogenic therapy in DME.

Images obtained with longer wavelength flash camera-based systems are correlated with OCT parameters in DME detection ${ }^{8,9}$ but have lower sensitivity in comparison with those obtained with shorter wavelengths. $^{\text {? }}$

Indices formed by the ratio of FAF grade and variables such as best-corrected visual acuity (BCVA) or macular volume (MV) might be useful as characteristics or baseline biomarkers that help predict the response to treatment with antiangiogenic therapy in patients with DME in comparison with variables not combined with FAF such as BCVA or baseline contrast sensitivity (CS).

The purpose of this study was to evaluate the correlations between FAF composite indices and other anatomical functional variables in patients with DME under treatment with ziv-aflibercept (ziv-AFL) intravitreal injections.

\section{Methods}

Retrospective sub-analysis of a series of cases previously published by our group. ${ }^{8,9}$ We included 29 eyes from 15 patients recruited at the Retina Department of Clínica David, Ophthalmology Unit, in the City of Morelia, Michoacán, Mexico.

Patients who received ziv-AFL intravitreal injections for the treatment of DME (defined as central foveal subfield $>260 \mu \mathrm{m}$ thickening) were included. Patients with any macular laser treatment (within a $1500-\mu \mathrm{m}$ diameter around the foveal center) or intraocular surgery performed within six months before the start of
ziv-AFL intravitreal injection treatment were excluded.

All patients underwent complete ophthalmological examination that included BCVA measurement, anterior segment examination, indirect ophthalmoscopy and biomicroscopy with 78D lens. FAF, OCT spectral domain images and posterior pole clinical photography were obtained.

\section{Procedures carried out during the study}

- All patients received three ziv-AFL intravitreal injections under sterile conditions the day of baseline evaluation, as well as at first and second month of follow-up. A previously described standardized method ${ }^{8-11}$ was used for the preparation of the injection site and for its application

- Far BCVA for each eye was measured with the Early Treatment Diabetic Retinopathy Study (ETDRS) standardized protocol at a distance of $4 \mathrm{~m}$, with a modified ETDRS far vision chart (Precision Vision, La Salle, Illinois, USA). CS was obtained with the Hamilton-Veale chart for CS evaluation (Hamilton Veale, Canterbury, New Zealand). Measurements of both BCVA and CS were obtained at baseline, as well as at first and second month

- Fundus autofluorescence. FAF images were obtained with a fundus camera (VISUCAM ${ }^{\circledR N M / F A}$, Carl Zeiss Meditec Inc., Oberkochen, Germany), with an excitation wavelength of 510 to $580 \mathrm{~nm}$ and emitted light detection above $640 \mathrm{~nm}$. FAF images were obtained at baseline and at first and second month

- The images were staged according to different foveal FAF patterns using a modified classification based on that described by Vujosevic et al. ${ }^{10}$ and Hernández-Da Mota et al. ${ }^{8-10}$ Classification grades were: grade 1 or decreased FAF (dFAF); grade 2 or normal FAF (nFAF); grade 3 or increased unifocal FAF; grade 4 or increased multifocal FAF and grade 5 or confluent or plaque-like multifocal increased FAF. Staging of the FAF images was carried out independently by two expert blinded evaluators

- OCT. Images were obtained with the Cirrus $^{\mathrm{TM}}$ HD-OCT 5000 equipment (Carl Zeiss Meditec Inc., Oberkochen, Germany), similarly as with the other variables (measurements were taken at baseline and at first and second month). 
The scanning protocol was that for macular thickness calculated based on the $512 \times 128$ macular cube program. For this study, the central foveal thickness (CFT) subfield value was obtained in microns $(\mu \mathrm{m})$. In addition, MV value was recorded in $\mathrm{mm}^{3}$, and that of mean macular thickness (MMT), in $\mu \mathrm{m}$.

\section{Statistical analysis}

All data were recorded and analyzed using the GraphPad Prism program, version 8 (GraphPad Software Inc., San Diego, California). Averages and their respective standard deviation were obtained for all baseline values.

FAF grade values were converted to their corresponding log values (grade $1=0$, grade $2=0.3$, grade $3=0.47$, grade $4=0.6$, grade $5=0.69$ ). ${ }^{9}$ Baseline indices were obtained by dividing baseline FAF log value by each one of the other baseline variables (BCVA measured in number of letters, CS measured in number of letter pairs, CFT measured in $\mu \mathrm{m}$, MV measured in $\mathrm{mm}^{3}$ and MMT measured in $\mu \mathrm{m}$ ).

In the correlation analysis, Spearman's rank correlation coefficients were obtained from baseline indices and different anatomical-functional variables during follow-up (first and second month).

Similarly, correlations were established between baseline variables values (BCVA, CS, CFT) and the values of variables (BCVA, MV and CS) obtained during follow-up. Linear regression analysis was applied, and inter-observer variability (kappa coefficient) was evaluated. Z-test was used to compare the different correlation coefficients that were obtained.

\section{Results}

Twenty-nine eyes from 15 patients were included in this case series. Patient age range was 53-74 years.

Baseline BCVA, CS, CFT, MV and MMT, as well as FAF patterns and macular edema OCT patterns of the patients included in the study are shown in table 1.

Inter-observer variability between the two evaluators for FAF staging was evaluated with the kappa coefficient $(0.806 ; p=0.000)$.

There were statistically significant correlations between baseline indices and some anatomical and functional variables during patient follow-up (Figs. 1-3). The strongest correlations were between FAF/BCVA baseline index and second month BCVA $\left(r_{s}=-0.78 ; p\right.$ $=0.0003$, Fig. 1), between FAF/CS baseline index and
Table 1. Baseline characteristics of the patients with DME included in the study

\begin{tabular}{|c|c|c|}
\hline $\begin{array}{l}\text { Age, years } \\
\text { Mean } \pm \text { SD } \\
\text { Range }\end{array}$ & \multicolumn{2}{|c|}{$\begin{array}{c}61.8 \pm 6.2 \\
53-74\end{array}$} \\
\hline BCVA, number of letters on ETDRS chart & \multicolumn{2}{|c|}{$32.3 \pm 16.3$} \\
\hline CS, number of letter pairs & \multicolumn{2}{|c|}{$6.8 \pm 3.7$} \\
\hline Central foveal thickness by OCT $(\mu \mathrm{m})$ & \multicolumn{2}{|c|}{$390 \pm 118.8$} \\
\hline Macular volume by OCT $\left(\mathrm{mm}^{3}\right)$ & \multicolumn{2}{|c|}{$11.2 \pm 3.2$} \\
\hline Mean macular thickness by OCT ( $\mu \mathrm{m})$ & \multicolumn{2}{|c|}{$383.8 \pm 95.7$} \\
\hline & $\mathrm{n}$ & $\%$ \\
\hline $\begin{array}{l}\text { Gender } \\
\text { Males } \\
\text { Females }\end{array}$ & $\begin{array}{c}10 \\
5\end{array}$ & $\begin{array}{l}75 \\
25\end{array}$ \\
\hline $\begin{array}{l}\text { FAF pattern } \\
\text { Grade } 1 \text { (decreased) } \\
\text { Grade } 2 \text { (normal) } \\
\text { Grade } 3 \text { (unifocal increased) } \\
\text { Grade } 4 \text { (multifocal increased) } \\
\text { Grade } 5 \text { (confluent or "plaque-like" multifocal } \\
\text { increased) }\end{array}$ & $\begin{array}{c}5 \\
11 \\
4 \\
6 \\
3\end{array}$ & $\begin{array}{l}17.2 \\
37.9 \\
13.7 \\
20.6 \\
10.3\end{array}$ \\
\hline $\begin{array}{l}\text { Edema pattern by OCT } \\
\text { Cystic } \\
\text { Non-cystic (spongiform) } \\
\text { Subfoveal serous neuroretinal detachment }\end{array}$ & $\begin{array}{c}7 \\
17 \\
5\end{array}$ & $\begin{array}{c}22.9 \\
60 \\
17.1\end{array}$ \\
\hline
\end{tabular}

BCVA: best-corrected visual acuity; CS: contrast sensitivity; OCT: optical coherence tomography; FAF: fundus autofluorescence.

first month MV $\left(r_{s}=0.64 ; p=0.001\right.$, Fig. 2) and between FAF/CFT baseline index and first month CS $\left(r_{s}=0.44 ; p=0.02 ;\right.$ Fig. 3).

The correlation between FAF/BCVA and FAF/CS baseline indices and MV at first month of follow-up was stronger than that observed between BCVA and CS baseline values and MV at first month of follow-up (Figs. 1 and 2). Table 2 shows the comparisons between the different correlation coefficients.

The comparison between baseline FAF/BCVA correlation with first month MV $\left(r_{s}=0.56\right)$ and baseline BCVA correlation with first month MV $\left(r_{s}=-0.1\right)$ was the one that showed statistical significance $(p=0.000$, table 2$)$.

\section{Discussion}

FAF has been described to be a useful method in the detection and follow-up of retinal diseases. ${ }^{6,8-12}$ However, a few studies have addressed the role of FAF in the evaluation of patients with DME. ${ }^{13-17}$

Calvo-Maroto et al. ${ }^{14}$ and Shen et al. ${ }^{15}$ described FAF findings and classification in DME. 
A

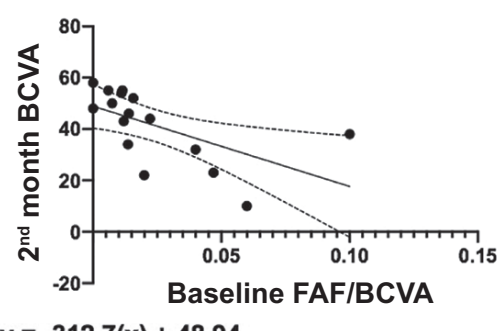

$y=-312.7(x)+48.94$

C

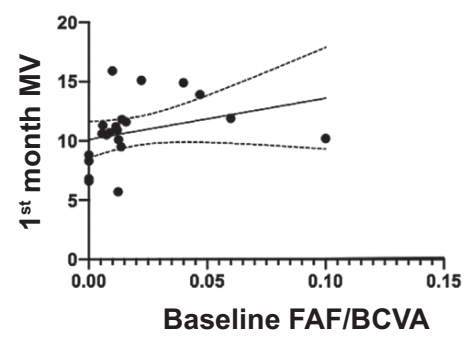

$y=34.89(x)+10.11$
B $r_{s}=0.8, p=0.0003,(95 \% \mathrm{Cl} 0.47-0.93)$

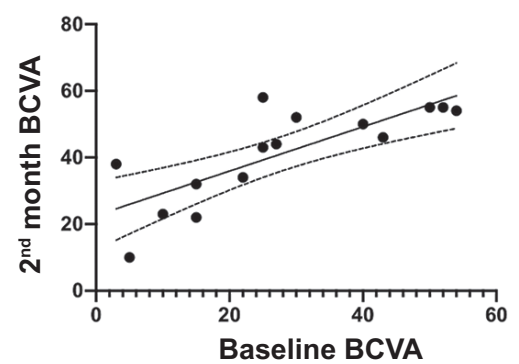

$y=0.6648(x)+22.63$

D $\quad r_{s}=-0.1, p=0.32,(95 \% \mathrm{Cl}-0.54-0.37)$

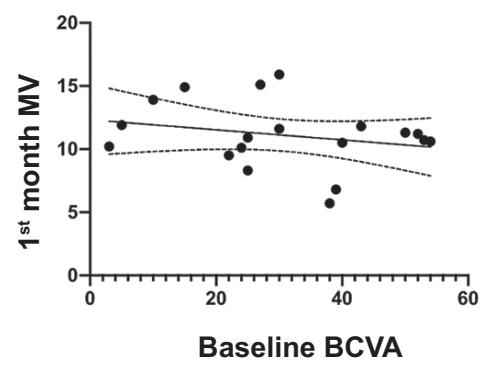

$y=-0.03990(x)+12.33$

Figure 1. (A) Correlation between fundus autofluorescence/best-corrected visual acuity (FAF/BCVA) baseline index and BCVA at second month of follow-up. (B) Correlation between FAF/BCVA baseline index and macular volume (MV) at 1 month of follow-up. (C) Correlation between baseline BCVA and BCVA at second month of follow-up. (D) Correlation between baseline BCVA and MV at first month.

A

$r_{s}=0.64, p=0.001(95 \% \mathrm{Cl} 0.27-0.85)$

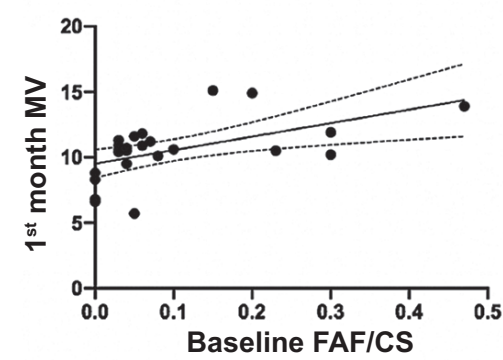

$y=10.33(x)+9.518$

C

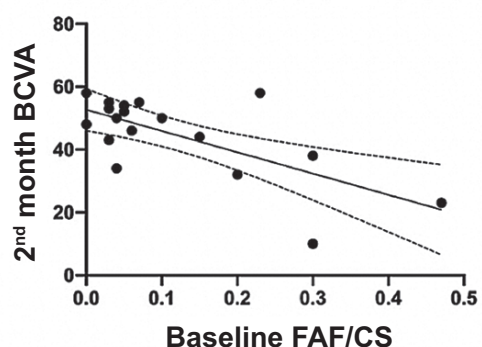

$y=-67.72(x)+52.7$
B $r_{s}=-0.2, p=0.17,(95 \% \mathrm{Cl}-0.63-0.27)$

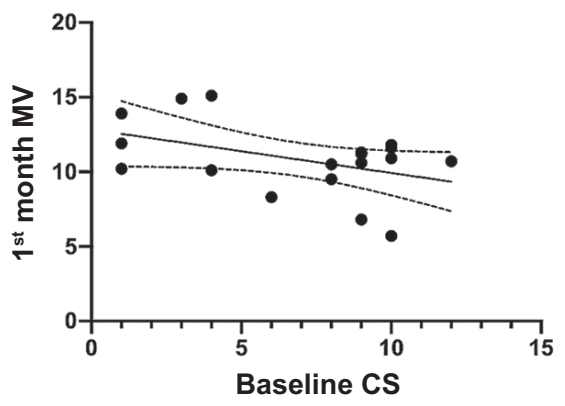

$y=-0.2910(x)+12.84$

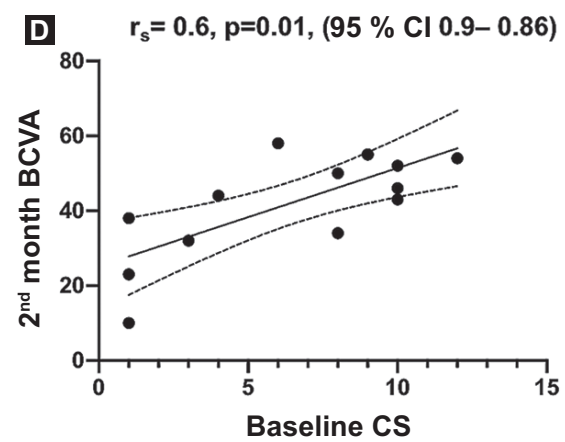

$y=2.624(x)+25.18$

Figure 2. (A) Correlation between fundus autofluorescence/contrast sensitivity (FAF/CS) baseline index and macular volume (MV) at first month of follow-up. (B) Correlation between FAF/CS baseline index and MV at $2^{\text {nd }}$ month of follow-up. (C) Correlation between baseline CS and MV at first month of follow-up. (D) Correlation between baseline CS and best-corrected visual acuity at second month. 
Table 2. Comparisons between correlations

\begin{tabular}{|c|c|c|c|c|}
\hline Correlation & $r_{s}$ & Correlation & $r_{s}$ & p-value \\
\hline Baseline FAF/BCVA-2 ${ }^{\text {nd }}$ month BCVA & -0.78 & Baseline BCVA-2 ${ }^{\text {nd }}$ month BCVA & 0.8 & 0.40 \\
\hline Baseline FAF/BCVA-1 $1^{\text {st }}$ month MV & 0.56 & Baseline BCVA-1st month MV & -0.1 & 0.000 \\
\hline Baseline FAF/CS- $1^{\text {st }}$ month MV & 0.64 & Baseline CS- $1^{\text {st }}$ month MV & -0.2 & 0.06 \\
\hline Baseline FAF/CS-2 ${ }^{\text {nd }}$ month BCVA & -0.61 & Baseline CS-2 ${ }^{\text {nd }}$ month BCVA & 0.6 & 0.48 \\
\hline Baseline FAF/CFT-2 $2^{\text {nd }}$ month MV & 0.40 & Baseline CFT-2 $2^{\text {nd }}$ month MV & -0.2 & 0.16 \\
\hline Baseline FAF/CFT- $1^{\text {st }}$ month CS & 0.44 & Baseline CFT-1 $1^{\text {st }}$ month CS & -0.5 & 0.37 \\
\hline
\end{tabular}

FAF: fundus autofluorescence; BCVA: best-corrected visual acuity; CS: contrast sensitivity; CFT: central foveal thickness; MV: macular volume.
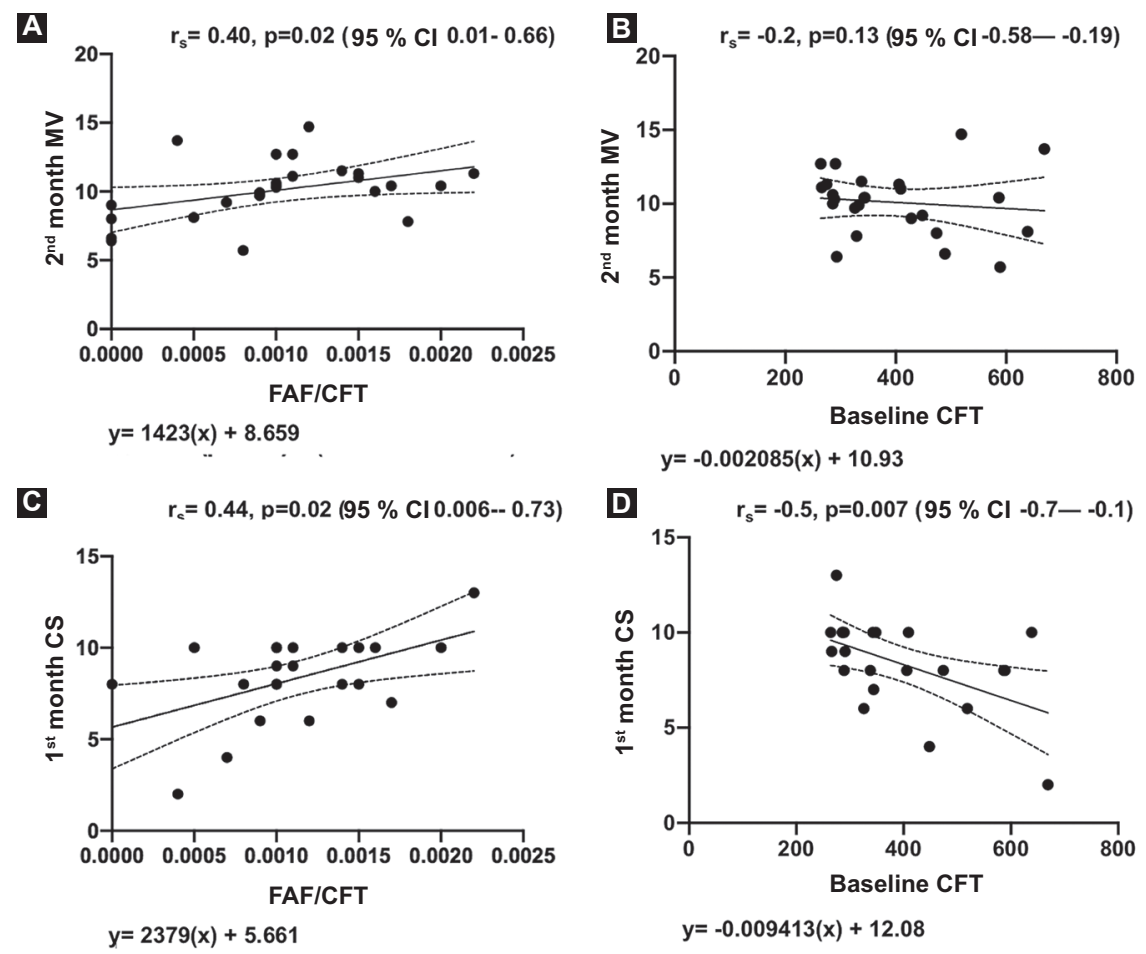

Figure 3. (A) Correlation between fundus autofluorescence/central foveal thickness (FAF/CFT) baseline index and macular volume (MV) at second month of follow-up. (B) Correlation between FAF/CFT baseline index and contrast sensitivity (CS) at first month of follow-up. (C) Correlation between baseline CFT and MV at second month of follow-up. (D) Correlation between baseline CFT and CS at first month.

This study indices that take FAF into account were correlated with anatomical-functional variables, such as some variables obtained on OCT, in order to determine the prediction of ziv-AFL treatment results in patients with DME. Similarly, comparisons were made between different correlations.

In a previous study, ${ }^{8}$ baseline FAF obtained in DME was shown to be positively correlated with structural changes on OCT. This correlation was able to predict short-term changes in macular thickness in patients under treatment with anti-VEGF therapy; however, the correlation was not as strong as that described in other publications. ${ }^{17}$

Frampton et al. ${ }^{7}$ showed that a longer wavelength in the acquisition of FAF images can translate into a lower sensitivity to detect alterations, which, in turn, might reduce the probability of detecting significant correlations with other variables. To compensate for this limitation with flash camera systems, in the present study, indices in which FAF grade was combined with other variables were employed. 
According to our results, FAF/CS and FAF/BCVA baseline indices had a strong negative correlation with BCVA in included patients' follow-up ( $r_{s}$ lower than -0.6). There was a strong positive correlation between FAF/CS baseline index and MV at follow-up first month $\left(r_{s}=0.64\right)$. Thus, FAF/BCVA and FAF/CS baseline indices could help to more accurately predict short-term BCVA (first and second months). Similarly, FAF/CS index might predict MV in patients under treatment with ziv-AFL for DME.

In a study previously published by our group, ${ }^{9}$ we suggested that there may be a potential benefit from applying some of the described indices as predictive biomarkers in patients with DME.

The FAF/BCVA index showed a significant correlation with BCVA at two months $\left(r_{s}=-0.78 ; p=0.0003\right)$. This correlation indicates that FAF baseline grade may be directly proportional to BCVA loss. Conversely, baseline BCVA is inversely proportional to BCVA at two months of treatment with ziv-AFL. ${ }^{9}$

The comparison between the correlation of baseline FAF/BCVA with MV at $1^{\text {st }}$ month $\left(r_{s}=0.56\right)$ and the correlation of baseline BCVA with MV at $1^{\text {st }}$ month $\left(r_{s}=-0.1\right)$ was the one that showed statistical significance. This suggests that the index formed by baseline FAF and BCVA would be a better baseline biomarker to predict $\mathrm{MV}$ reduction in response to zivAFL treatment than baseline BCVA.

Pece et al..$^{18}$ carried out qualitative correlations of FAF images in diabetic CME with FAF and OCT findings. They concluded that, with the confocal scanning laser imaging system that employs a short excitatory wavelength $(488 \mathrm{~nm})$, multilobulated spaces with increased autofluorescence can be visualized in patients with CME.

Reznicek et al. $^{12}$ evaluated green-light FAF, also in DME. Their results indicated that there were no significant correlations between FAF intensity and CFT, ellipsoid layer integrity, and RPE-ellipsoid layer thickness in diabetic patients with and without DME. Conversely, in our results, with a longer excitatory wavelength, statistically significant correlations were shown using FAF grade-containing composite indices.

Correlations in our study are consistent with those reported by Chung et al. ${ }^{17}$ FAF measurements correlated well with quantitative variables $(r=0.7)$. They also determined that associations could predict ellipsoid layer integrity restoration with a subsequent improvement in visual capacity.

On the other hand, Vujosevic et al. ${ }^{11}$ determined that FAF correlated better with central field microperimetry and OCT patterns than with BCVA. Average retinal sensitivity in areas with increased FAF was found to be decreased with regard to areas with FAF normal pattern. MMT and MV showed no significant differences between FAF different patterns. A moderately strong correlation was found between FAF/CS baseline index and MV at one month of follow-up $\left(r_{s}=0.64\right)$.

Another difference in our study with regard to that by Vujosevic et al ${ }^{10}$ was that these authors classified decreased FAF as part of FAF normal pattern, whereas we classified it as an independent pattern, staging it as grade $1 \mathrm{FAF}^{8}$

In another investigation, ${ }^{16}$ changes in specific imaging biomarkers (hyper-reflective intra-retinal spots and increased FAF) were evaluated as potential indicators of retinal inflammation. A negative correlation was found between baseline FAF (increased) and BCVA in eyes treated with bevacizumab, also in DME $\left(r_{s}=-0.21\right)$. In our study, this negative correlation was even stronger between FAF/BCVA baseline index and second month BCVA $\left(r_{s}=-0.78\right)$, as well as between FAF/CS baseline index and BCVA, also at second month $\left(r_{s}=-0.61\right)$.

Our research has some limitations. Included patients had a follow-up of only 2 months. On the other hand, the use of flash camera systems to obtain FAF images can increase the risk of scattered light uptake, which produces a false FAF increase, a phenomenon known as pseudo-autofluorescence. In addition, with flash cameras, low-contrast images are obtained, which can lead to FAF patterns misinterpretation. ${ }^{6}$ Finally, there is a lack of standardization and normative database for FAF; therefore, FAF is currently not considered a screening method of choice for numerous retinal diseases. Only a few studies ${ }^{17}$ have systematically reviewed FAF measurements reproducibility and consistency.

\section{Conclusions}

Indices such as baseline FAF/BCVA or baseline FAF/CS could be more useful as predictive biomarkers of the response to this treatment than FAF grade alone in patients with DME.

In this sense, the combination of two baseline variables in an index could provide an important synergistic effect for the prediction of variables of anatomical or functional response to antiangiogenic treatment in these patients, at least in the short-term.

\section{Acknowledgments}

The authors wish to thank the entire technical personnel at Clínica David and Sanatorio de La Luz of the city of Morelia, Michoacán, Mexico. 


\section{Conflict of interests}

The authors declare that they have no conflicts of interest.

\section{Funding}

The authors declare that they have no financial interest or participation in the self-funding of the project by Clínica David, Ophthalmology Unit, in the City of Morelia, Michoacán.

\section{Ethical disclosures}

Protection of human and animal subjects. The authors declare that no experiments were performed on humans or animals for this research.

Confidentiality of data. The authors declare that they followed the protocols of their work center on the publication of patient data.

Right to privacy and informed consent. The authors declare that no patient data appear in this article.

\section{References}

1. Klein R, Knudtson MD, Lee KE, Gangnon R, Klein BE. The Wisconsin epidemiologic study of diabetic retinopathy XXIII: the twenty-five-year incidence of macular edema in persons with Type 1 diabetes. Ophthalmology. 2009:116:497-503.

2. Shaw JE, Sicree RA, Zimmet PZ. Global estimates of the prevalence of diabetes for 2010 and 2030. Diabetes Res Clin Pract. 2010;87:4-14.

3. Klein R, Klein BE, Moss SE, Davis MD, DeMets DL. The Wisconsin epidemiologic study of diabetic retinopathy. IV: diabetic macular edema. Ophthalmology. 1984;91:1464-74.
4. Midena E, Bini S. Multimodal retinal imaging of diabetic macular edema: toward new paradigms of pathophysiology. Graefes Arch Clin Exp Ophthalmol. 2016;254:1661-8.

5. Acon D, Wu L. Multimodal imaging in diabetic macular edema. Asia Pac J Ophthalmol (Phila). 2018;7:22-7.

6. Yung M, Klufas MA, Sarraf D. Clinical applications of fundus autofluorescence in retinal disease. Int J Retina Vitreous. 2016;2:12.

7. Frampton GK, Kalita N, Payne L, Colquitt JL, Loveman E, Downes SM, et al. Fundus autofluorescence imaging: systematic review of test accuracy for the diagnosis and monitoring of retinal conditions. Eye (Lond). 2017:31:995-1007.

8. Hernández-Da Mota SE, Melo-Granados EA, Fromow-Guerra J, Bejar-Cornejo F, Gallego-Pinazo R, Rodríguez-Ayala E. Correlation analysis of fundus autofluorescence, spectral domain optical coherence tomography, and visual function in patients with diabetic macular oedema treated with intravitreal ziv-aflibercept. Eur J Ophthalmol. 2019;29:271-7.

9. Hernández-Da Mota SE, Béjar-Cornejo F, Esquivel-Velázquez M, Lima-Gómez V, González-Saldívar G, Rodríguez-Ayala E, et al. Autofluorescence indexes as biomarkers for antiangiogenic loading dose outcome in diabetic macular edema. Ther Adv Ophthalmol. 2020;12:2515841420942662.

10. Vujosevic S, Casciano M, Pilotto E, Boccassini B, Varano M, Midena E. Diabetic macular edema: fundus autofluorescence and functional correlations. Invest Ophthalmol Vis Sci. 2011;52:442-8.

11. Henández-Da Mota SH, Lima-Gómez V Rodríguez-Ayala E, Fromow-Guerra JJ, Melo-Granados EA. Classification of fundus autofluorescence abnormal patterns in diabetic macular edema. Gac Med Mex. 2019;155:458-62

12. Reznicek L, Dabov S, Haritoglou C, Kampik A, Kernt M, Neubauer AS. Green-light fundus autofluorescence in diabetic macular edema. Int $J$ Ophthalmol. 2013;6:75-80.

13. Costa-de Andrade G, de Oliveira-Dias JR, Maia A, Farah ME, Mitne S, Büchele-Rodrigues E. Intravitreal injections of Ziv-aflibercept for diabetic macular edema: a pilot study. Retina. 2016;36:1640-5.

14. Calvo-Maroto AM, Pérez-Cambrodi RJ, García-Lázaro S, Ferrer-Blasco T, Cerviño A. Ocular autofluorescence in diabetes mellitus. A review. J Diabetes. 2016;8:619-28.

15. Shen $Y, X u X$, Liu K. Fundus autofluorescence characteristics in patients with diabetic macular edema. Chin Med J (Engl). 2014; 127:1423-8.

16. Vujosevic S, Torresin T, Bini S, Convento E, Pilotto E, Parrozzani R, et al. Imaging retinal inflammatory biomarkers after intravitreal steroid and anti-VEGF treatment in diabetic macular edema. Acta Ophthalmol. 2017:95:464-71.

17. Chung H, Park B, Shin HJ, Kim HC. Correlation of fundus autofluorescence with spectral-domain optical coherence tomography and vision in diabetic macular edema. Ophthalmology. 2012;119:1056-65.

18. Pece A, Isola V, Holz F, Milani P, Brancato R. Autofluorescence imaging of cystoid macular edema in diabetic retinopathy. Ophthalmologica. 2010;224:230-5 